${ }^{1}$ Facultad de Medicina Universidad de Desarrollo -

Clínica Alemana de Santiago. Santiago, Chile.

${ }^{2}$ Servicio de Imagenología. Hospital Militar de Santiago. Santiago, Chile.

${ }^{3}$ Departamento de Hematooncología. Hospital Militar de

Santiago. Santiago, Chile. ${ }^{4}$ Servicio de Anatomía Patológica. Hospital Militar de Santiago. Santiago, Chile.

Trabajo no recibió financiamiento. Los autores declaran no tener conflictos de interés.

Recibido el 23 de junio de 2020, aceptado el 3 de marzo de 2021

Correspondencia a: Dr. Giancarlo Schiappacasse Faúndes

Av. Alcalde Fernando Castillo Velasco 9100. La Reina. gschiappacasse@gmail.com

\section{Metástasis ganglionar mesentérica de neoplasia neuroendocrina: una causa infrecuente de obstrucción de intestino delgado}

\author{
GIANCARLO SCHIAPPACASSE ${ }^{1,2}$, SOFÍA PALACIOS ${ }^{2}$, \\ BEATRIZ COMPARINI ${ }^{3}$, RENATO SANDOVAL ${ }^{4}$
}

\section{Small bowel obstruction caused by lymph nodes of a neuroendocrine tumor. A clinical case report}

\begin{abstract}
Small intestine neuroendocrine tumors (NET-SI) are relatively rare neoplasms. If encountered, the most common location is the ileum. Symptoms are usually non-specific, delaying the tumors diagnosis. NET-SI are often small in size and can be challenging to recognize on imaging studies. However, they have a tendency to induce a pronounced fibrotic reaction in the mesentery, often accompanied by large calcified mesenteric adenopathies. In some cases, the fibrotic reaction can produce rare complications, such as intestinal obstruction or vascular congestion with occasional secondary ischemia. This case report presents a 79-year-old male with a partial small bowel obstruction caused by a fibrotic reaction and mesenteric adenopathies of a well-differentiated neuroendocrine tumor of the ileum. The patient also presented multiple peritoneal metastases at diagnosis. Characteristic imaging findings of the tumor, allowed an accurate and early diagnosis. Once the acute episode was resolved, the diagnosis was confirmed with an image guided biopsy.
\end{abstract}

(Rev Med Chile 2021; 149: 464-468)

Key words: Ileal Neoplasms; Intestinal Obstruction; Intestine, Small; Lymphadenopathy; Neuroendocrine Tumors.
L os tumores neuroendocrinos de intestino delgado (TNE-ID) son tumores epiteliales en que predomina la diferenciación neuroendocrina ${ }^{1}$. Los TNE-ID constituyen un grupo heterogéneo de neoplasias infrecuentes de lento crecimiento, las cuales presentan algunas características comunes a todas las formas de tumores neuroendocrinos y otras particulares del órgano desde donde se originan.

Los TNE-ID de yeyuno e íleon suelen tener un comportamiento y forma de presentación diferente a los localizados en duodeno, presentándose habitualmente en la sexta y séptima década y sin preferencia de género. La mayor parte de los TNE-ID de íleon son tumores no funcionantes y los pacientes suelen presentar síntomas tardíos, cuando ya existen metástasis locorregionales o a distancia ${ }^{1-3}$.

Las localizaciones más frecuentes de diseminación son los linfonodos locorregionales, el mesenterio e hígado; hasta $65 \%$ de los pacientes presenta diseminación a una o más de estas localizaciones al momento del diagnóstico ${ }^{2,4,5}$.

La sobrevida a 5 años se correlaciona directamente con la etapa al momento del diagnóstico, siendo $65 \%$ para pacientes con enfermedad loca- 
lizada y solo $36 \%$ para aquellos con metástasis a distancia ${ }^{6}$.

Las manifestaciones clínicas iniciales incluyen dolor abdominal difuso, diarrea, baja de peso y, en casos menos habituales, pueden debutar como obstrucción intestinal intermitente o sangrado ${ }^{1,3}$.

\section{Caso clínico}

Paciente de sexo masculino de 79 años, con antecedente de hipertensión arterial, resistencia a la insulina, hipercolesterolemia y prostatectomía radical.

Consultó en el servicio de urgencia por un cuadro clínico de 3 días de evolución caracterizado por dolor abdominal difuso, diarrea crónica, náuseas y vómitos. $\mathrm{Al}$ examen físico presentaba abdomen distendido y dolor abdominal difuso.

Se realizó una tomografía computada (TC) de abdomen y pelvis con contraste endovenoso, que demostró una lesión nodular de comportamiento hipervascular de $22 \mathrm{~mm}$ de diámetro, localizada a nivel del íleon distal. Se identificaron, además, dos grandes lesiones sólidas espiculadas con calcificaciones centrales, localizadas en mesenterio, las que generaban una importante reacción desmoplásica
(Figura 1); la de localización más caudal medía $55 \mathrm{~mm}$ y la otra ubicada en la raíz del mesenterio medía $35 \mathrm{~mm}$ de diámetro mayor. Ambas lesiones determinaban una importante congestión de los vasos mesentéricos con zonas de dilatación de yeyuno e íleon que se asociaban a edema de la submucosa y congestión de la vasa recta, sin signos de necrosis, perforación ni colecciones asociadas. Se identificaron, además, varios nódulos a nivel del omento mayor con características de implantes secundarios.

Los hallazgos descritos eran altamente sugerentes de una neoplasia neuroendocrina de intestino delgado localizada en íleon distal con diseminación peritoneal secundaria y adenopatías mesentéricas con calcificaciones centrales, que producían una reacción fibrótica/desmoplásica con retracción de las asas de intestino delgado, determinando isquemia venosa y una obstrucción intestinal parcial.

Se decidió realizar manejo médico de la obstrucción intestinal parcial con resolución del cuadro agudo y cese de los síntomas. De manera diferida, el equipo de radiología intervencional realizó una biopsia de los implantes omentales de aspecto secundario. El resultado histológico confirmó el diagnóstico de un tumor neuroendocrino, que en base a la clasificación de la World Health

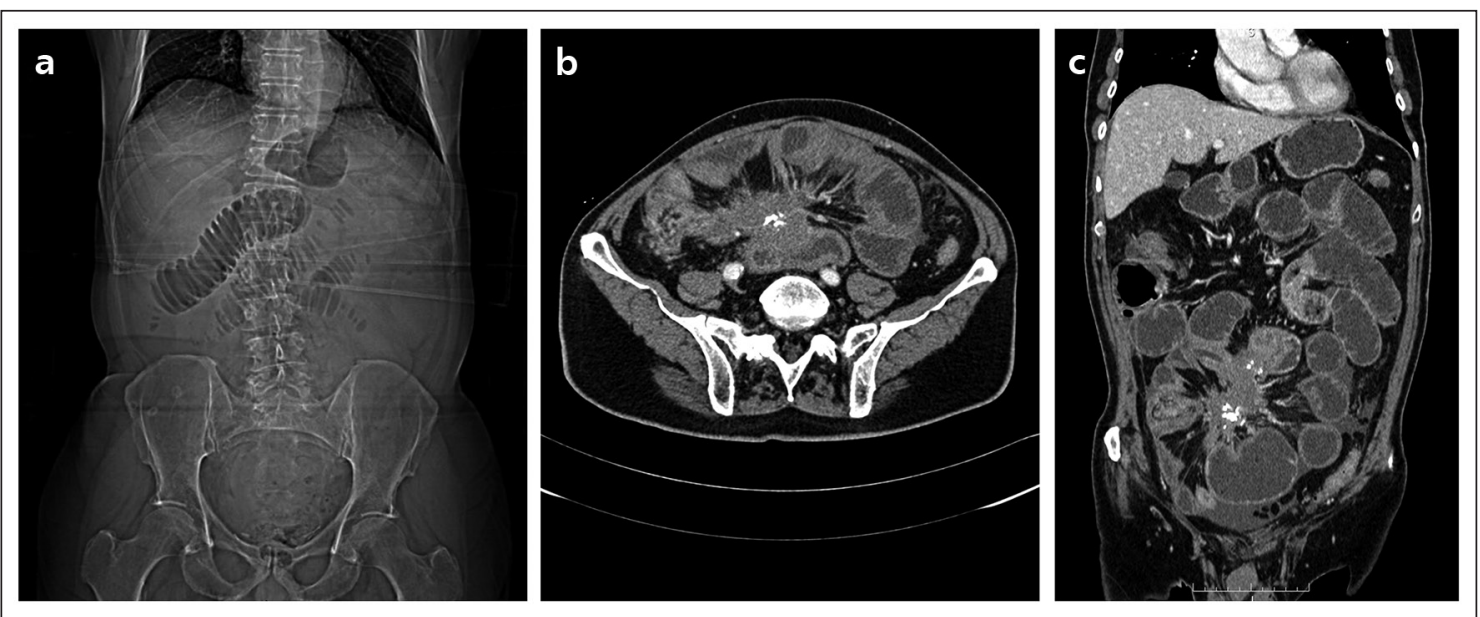

Figura 1. TNE ileal en paciente masculino de 79 años con dos masas ganglionares mesentéricas que determinan fibrosis local, congestión venosa y obstrucción intestinal parcial secundaria de delgado. (a) Topograma de TC de abdomen y pelvis que demuestra moderada dilatación de algunas asas de intestino delgado con patrón en "pila de monedas". (b) y (c) TC de abdomen y pelvis con contraste intravenoso en fase porto-venosa en los planos axial y coronal respectivamente, que evidencian masas mesentéricas parcialmente calcificadas rodeadas de una reacción desmoplásica de aspecto "radiado". 


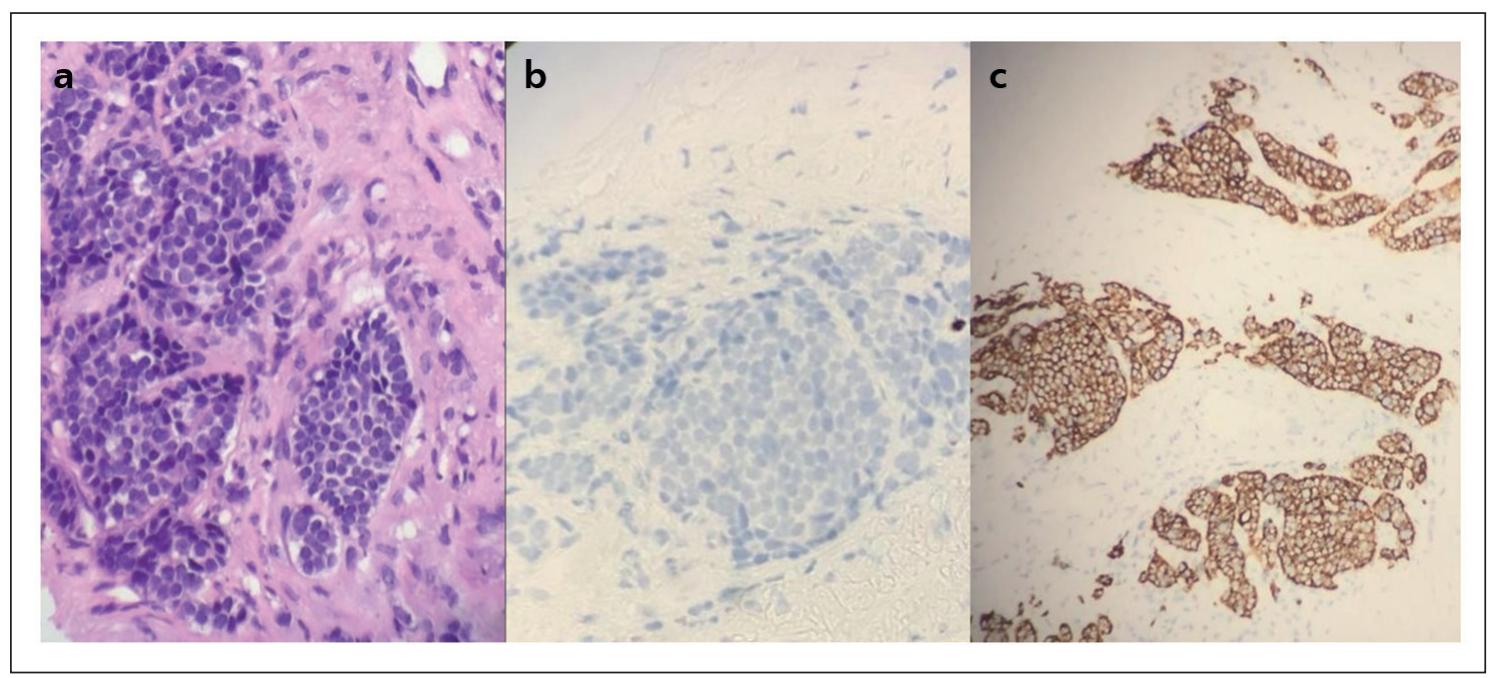

Figura 2. Histopatología de biopsia de TNE con (a) Tención con hematoxilina-eosina (b) Tinción difusa con cromogranina y (c) Tinción difusa con sinaptofisina. Se aprecian nidos con desarrollo de empalizada periférica y rodeados de un estroma fibroso; están conformados por células relativamente homogéneas de núcleos pequeños, con índice mitótico bajo (inferior al $1 \%$ ) y citoplasma escaso. Tinción para Ki-67 revela un índice proliferativo menor al 1\% de las células (tinción nuclear), lo que es concordante con un TNE de bajo grado - G1 (bien diferenciado).

Organization (WHO) de $2019^{5}$, correspondía a un tumor bien diferenciado.

En forma complementaria, se realizó una PET-CT con $\mathrm{Ga}^{68}$-Dotatate que mostró aumento de la captación con el radiofármaco a nivel de las lesiones previamente descritas, confirmando los hallazgos previamente descritos.

Con estos resultados se realizó una evaluación por el comité oncológico multidisciplinario y se decidió iniciar tratamiento con el inhibidor multicinasa sunitinib (Sutent ${ }^{\circledR}$, Pfizer, Inc. NY, EE.UU.) y el agente somatostático ocreotide (Sandostatin LAR $^{\circledR}$, Novartis).

Actualmente, el paciente se mantiene estable, en controles habituales y sin signos de complicación.

\section{Discusión}

La incidencia anual de TNE-ID ha aumentado durante los últimos años, alcanzando aproximadamente 40-50 casos por millón de habitantes. Se cree que este aumento en la incidencia se debe a mayor número y mejores métodos diagnósticos, además de una mayor disponibilidad de estos, más que a un incremento real de la incidencia ${ }^{1,6,7}$.

El mayor porcentaje de los TNE-ID son de grado intermedio o bajo y presentan crecimiento local lento. Sin embargo, frecuentemente se realiza el diagnóstico cuando se encuentran en etapas avanzadas de diseminación ${ }^{1,8}$.

Los TNE-ID presentan un pronóstico habitualmente menos favorable en comparación con tumores de similar tamaño en otras localizaciones, lo que, probablemente, se debe a que presentan mayor tendencia a crecer y metastatizar antes de que se logre el diagnóstico ${ }^{1}$.

Afortunadamente, la evaluación con TC multifásica de alta resolución junto a métodos de postproceso (como reconstrucciones multiplanares) representan una herramienta muy valiosa para lograr el diagnóstico de manera precoz ${ }^{4}$. Habitualmente, los tumores primarios son pequeños $y$, usualmente, de considerable menor tamaño que la enfermedad mesentérica y ganglionar secundaria. En casos, en que el tumor no es notoriamente hipervascular, puede ser muy difícil de evaluar en un intestino poco distendido ${ }^{9-11}$. El engrosamiento focal de la pared intestinal debe hacer sospechar patología focal y obliga a una evaluación dirigida de este segmento. 
A pesar de lo anteriormente mencionado, incluso en enterografía por TC (examen dirigido a la evaluación de intestino delgado distendido luego de la administración de medio de contraste negativo como el agua) y en pacientes con adecuada preparación, hasta $14 \%$ de los tumores menores a $10 \mathrm{~mm}$ pueden pasar inadvertidos ${ }^{12}$.

El diagnóstico por imágenes de los TNE-ID de pequeño tamaño no es raro que se realice en base a las manifestaciones por diseminación secundaria del tumor. Las principales manifestaciones descritas son: compromiso ganglionar locorregional y al mesenterio y metástasis hematógenas a distancia.

La diseminación ganglionar mesentérica, como en el caso descrito anteriormente, produce característicamente una importante reacción desmoplásica en su periferia, otorgando un aspecto de "retracción" en las hojas del mesenterio. La reacción desmoplásica refleja fibrosis, pero también puede representar infiltración difusa por células tumorales. Las adenopatías secundarias se encuentran parcialmente calcificadas hasta en 70\% de los casos y pueden, además, presentar realce ávido con el medio de contraste intravenoso ${ }^{4,9,13}$.

La reacción desmoplásica, ocasionalmente, puede producir complicaciones como torsión o retracción de la pared intestinal con obstrucción de la irrigación, inicialmente venosa y eventualmente arterial, pudiendo derivar en una isquemia secundaria del segmento comprometido $y$, además, causar obstrucción ${ }^{15-19}$.

La identificación de fibrosis mesentérica de aspecto "estriado" o "radiado" rodeando una masa mesentérica parcialmente calcificada, y que no se continua directamente con la pared intestinal, es prácticamente un hallazgo patognomónico para metástasis ganglionar de un tumor neuroendocrino $^{9,19}$.

Respecto a los diagnósticos diferenciales, se puede considerar al linfoma tratado y a la mesenteritis retráctil o esclerosante. Sin embargo, ambas suelen diferenciarse en base a la historia clínica, otros hallazgos imagenológicos y exámenes de laboratorio ${ }^{4}$.

En resumen, se presenta un caso clínico que representa una causa infrecuente de obstrucción intestinal, pero que debe ser sospechada dada la presentación característica en imágenes de este tipo de lesiones. Esto permite un diagnóstico más precoz y un manejo adecuado de los pacientes.

\section{Referencias}

1. Xavier S, Rosa B, Cotter J. Small bowel neuroendocrine tumors: From pathophysiology to clinical approach. World J Gastrointest Pathophysiol. 2016; 7 (1): 117.

2. Modlin IM, Sandor A. An analysis of 8305 cases of carcinoid tumors. Cancer. 1997; 79 (4): 813-29.

3. Chang S, Choi D, Soon JL, Won JL, Park MH, Sang WK, et al. Neuroendocrine neoplasms of the gastrointestinal tract: Classification, pathologic basis, and imaging features. Radiographics 2007 Nov 1; 27 (6): 1667-79.

4. Bonekamp D, Raman SP, Horton KM, Fishman EK. Role of computed tomography angiography in detection and staging of small bowel carcinoid tumors. World J Radiol. 2015; 7 (9): 220.

5. Nagtegaal ID, Odze RD, Klimstra D, Paradis V, Rugge M, Schirmacher P, et al. The 2019 WHO classification of tumours of the digestive system. Histopathology 2020; 76 (2): 182-8.

6. Eriksson B, Klöppel G, Krenning E, Ahlman H, Plöckinger U, Wiedenmann B, et al. Consensus guidelines for the management of patients with digestive neuroendocrine tumors - Well-differentiated jejunal-ileal tumor/carcinoma. Neuroendocrinology 2007; 87 (1): 8-19.

7. Fitzgerald TL, Dennis SO, Kachare SD, Vohra NA, Zervos EE. Increasing incidence of duodenal neuroendocrine tumors: Incidental discovery of indolent disease? Surgery 2015; 158 (2): 466-71.

8. Cho MY, Kim JM, Sohn JH, Kim MJ, Kim KM, Kim $\mathrm{WH}$, et al. Current trends of the incidence and pathological diagnosis of gastroenteropancreatic neuroendocrine tumors (GEP-NETs) in Korea 2000-2009: Multicenter study. Cancer Res Treat. 2012; 44 (3): 157-65.

9. Pantongrag-Brown L, Buetow PC, Carr NJ, Lichtenstein JE, Buck JL. Calcification and fibrosis in mesenteric carcinoid tumor: CT findings and pathologic correlation. Am J Roentgenol. 1995; 164 (2): 387-91.

10. Picus D, Glazer HS, Husband JE. Computed Abdominal Tomography of Carcinoid Tumors. Am J Roentgenol.1984; 143 (3): 581-4

11. Soyer P, Boudiaf M, Fishman EK, Hoeffel C, Dray X, Manfredi R, et al. Imaging of malignant neoplasms of the mesenteric small bowel: New trends and perspectives. Crit Rev Oncol Hematol. 2011; 80 (1): 10-30.

12. Datta S, Williams N, Suortamo S, Mahmood A, Oliver C, Hedley N, et al. Carcinoid syndrome from small bowel endocrine carcinoma in the absence of hepatic metastasis. Age Ageing 2011; 40 (6): 760-2.

13. Hoeffel C, Crema MD, Belkacem A, Azizi L, Lewin M, 
Arrivé L, et al. Multi-detector row CT: spectrum of diseases involving the ileocecal area. Radiographics 2006; 26 (5): 1373-90.

14. Vijayakumar HM, Kalaivani V, Kedage V, Subbaraya S. Case Report A Rare Cause for Sub-acute Small Bowel Obstruction: Distal Ileal Neuroendocrine Tumor. IJSS Journal of Surgery 2016; 2 (12): 45-7.

15. Prasad ND, Manmadharao V, Babji K, Reddy DK. Intestinal Obstruction by Carcinoid Tumor in Ileum: A Case Report. Int J Sci Stud 2015; 2 (10): 166-8.

16. Selberherr A, Niederle MB, Niederle B. Surgical Treatment of Small Intestinal Neuroendocrine Tumors G1/
G2. Visc Med. 2017; 33 (5): 340-3.

17. Aleksandar R, Vladimir R, Miroslav D, Arafeh M, Aleksic A, Gojgic M. A Rare Case of a Small Bowel Obstruction Caused by a Ileal Neuroendocrine Tumor. M J Surg. 2017; 1 (2): 8-10.

18. Moosavy SH, Andrabi Y, Esmaeeli S, Amini A. Small bowel obstruction by a terminal ileum carcinoid tumor: a case report. MJIRI 2011; 25 (3): 165-9.

19. Sheth S, Horton KM, Garland MR, Fishman EK. Mesenteric Neoplasms: CT Appearances of Primary and Secondary Tumors and Differential Diagnosis. Radiographics. 2003; 23 (2): 457-73. 